\title{
The influence of culture-dependent native microbiota in Zika virus infection in Aedes aegypti
}

\author{
Rêgila Mello do Nascimento ${ }^{1,2,3 \dagger}$, Thais Bonifácio Campolina ${ }^{1,4+}$, Barbara Aparecida Chaves², \\ Jessica Lana Fernandes Delgado', Raquel Soares Maia Godoy', Paulo Filemon Paolucci Pimenta 1,3,4 and \\ Nagila Francinete Costa Secundino $1,3,4^{*}$ (D)
}

\begin{abstract}
Background: Emerging and re-emerging vector-borne diseases (VBDs) pose a recurring threat to tropical countries, mainly due to the abundance and distribution of the Aedes aegypti mosquito, which is a vector of the Zika, dengue, chikungunya, and yellow fever arboviruses.

Methods: Female 3-5 day-old Ae. aegypti were distributed into two experimental groups: group I—survey of cultivable bacteria; sucrose group: fed only on sucrose, i.e., non-blood-fed (UF); blood-fed group: (i) fed with non-infected blood (BF); (ii) fed with blood infected with the Zika virus (BZIKV); (iii) pretreated with penicillin/streptomycin (pen/ strep), and fed with non-infected blood (TBF); (iv) pretreated with pen/strep and fed blood infected with ZIKV, i.e., gravid with developed ovaries, (TGZIKV); group II-experimental co-infections: bacteria genera isolated from the group fed on sucrose, i.e., non-blood-fed (UF).

Results: Using the cultivable method and the same mosquito colony and ZIKV strain described by in a previous work, our results reveled 11 isolates (Acinetobacter, Aeromonas, Cedecea, Cellulosimicrobium, Elizabethkingia, Enterobacter, Lysinibacillus, Pantoea, Pseudomonas, Serratia, and Staphylococcus). Enterobacter was present in all evaluated groups (i.e., UF, BF, BZIKV, TBF, and TGZIKV), whereas Elizabethkingia was present in the UF, BZIKV, and TBF groups. Pseudomonas was present in the BZIKV and TBF groups, whereas Staphylococcus was present in the TBF and TGZIKV groups. The only genera of bacteria that were found to be present in only one group were Aeromonas, Lysinibacillus, and Serratia (UF); Cedacea, Pantoea and Acinetobacter (BF); and Cellulosimicrobium (BZIKV). The mosquitoes co-infected with ZIKV plus the isolates group fed on sucrose (UF) showed interference in the outcome of infection.

Conclusions: We demonstrate that the distinct feeding aspects assessed herein influence the composition of bacterial diversity. In the co-infection, among ZIKV, Ae. aegypti and the bacterial isolates, the ZIKV/Lysinibacillus-Ae. aegypti had the lowest number of viral copies in the head-SG, which means that it negatively affects vector competence. However, when the saliva was analyzed after forced feeding, no virus was detected in the mosquito groups ZIKV/Lysinibacillus-Lu. longipalpis and Ae. aegypti; the combination of ZIKV/Serratia may interfere in salivation. This
\end{abstract}

\footnotetext{
*Correspondence: secundinon@gmail.com

${ }^{\dagger}$ Rêgila Mello do Nascimento and Thais Bonifácio Campolina are equally contributing first authors

1 Laboratorio de Entomologia Médica, Instituto René Rachou-FIOCRUZMinas, Belo Horizonte, Minas Gerais, Brazil

Full list of author information is available at the end of the article
}

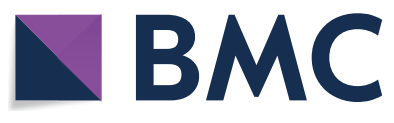

Then Access This article is licensed under a Creative Commons Attribution 4.0 International License, which permits use, sharing, adaptation, distribution and reproduction in any medium or format, as long as you give appropriate credit to the original author(s) and the source, provide a link to the Creative Commons licence, and indicate if changes were made. The images or other third party material in this article are included in the article's Creative Commons licence, unless indicated otherwise in a credit line to the material. If material is not included in the article's Creative Commons licence and your intended use is not permitted by statutory regulation or exceeds the permitted use, you will need to obtain permission directly from the copyright holder. To view a copy of this licence, visit http://creativecommons.org/licenses/by/4.0/. The Creative Commons Public Domain Dedication waiver (http://creativeco mmons.org/publicdomain/zero/1.0/) applies to the data made available in this article, unless otherwise stated in a credit line to the data. 
indicates that the combinations do not produce viable viruses and may have great potential as a method of biological control.

Keywords: Cultivable bacteria, Microbiota, Co-culture, Zika virus

\section{Background}

Emerging and re-emerging vector-borne diseases (VBDs) pose a recurring threat to tropical and subtropical countries. In terms of their impact on public health and epidemiology, VBDs are responsible for $17 \%$ of all infectious diseases globally, which result in around 700,000 deaths per year [1]. The intensification of arbovirus transmission is mainly due to the abundance and distribution of the Aedes aegypti mosquito, a main vector of the Zika (ZIKV), dengue (DENV), chikungunya (CHIKV), and urban yellow fever arboviruses. In Brazil, despite regular vector control programs, Ae. aegypti persists in all states and has high urban density [2].

The principal approach for vector control of arboviruses is chemical or biological control. Chemical control focuses on the identification and elimination of breeding sites and adults $[3,4]$. Biological control, i.e., the use of natural enemies, traditionally includes intervention in breeding sites. New strategies for vector control include the insecticide pyriproxyfen (PPF), which can be delivered by the insect and has the potential to block viral transmission, even in adverse scenarios [5], and the use of genetically modified endosymbionts (paratransgenesis) [6] and transgenic mosquitoes, such as the Ae. Aegypti strain (OX513A), which is genetically modified to prevent their offspring from developing into adults [7].

In the last few decades, advances in the study of the insect-microbiota relationship have indicated the possibility of using elements of the microbiota in vector control, with the aim of altering or using taxa with the capacity to influence the host's physiology $[8,9]$. One pioneering and successful study using this technique involved Rhodococcus rhodnii, an endosymbiont of Rhodnius prolixus and a vector of Chagas disease, which was modified to express cecropin A. This peptide results in the elimination or reduction of the number of Trypanosoma cruzi in the vector [10].

More recently, the bacteria of the genus Asaia, which was isolated from Anopheles stephensi, was modified to express and secrete anti-Plasmodium molecules capable of interfering with the activity of Plasmodium berghei, resulting in a significant reduction in the development of oocysts $[11,12]$ and a reduction in the number of pathogens ingested in the gut of insect vectors even before the end of the digestive process. Different factors are responsible for this initial reduction, such as digestive enzymes or innate response. Another possible explanation could be the action of the intracellular or extracellular bacteria in the digestive tract. Inside the midgut, pathogens and extracellular bacteria will be interacting for the first time, both competing for nutrients and survival, mainly with extracellular bacteria. As an example of intracellular bacteria, the bacterium Wolbachia is a known ally for VBDs. It stands out due to its ability to spread through insect populations and its effect on vector competence [13, 14].

In Culicidae, the intestinal microbiota is essential for the development and survival of the immature insect forms [15]. In addition, studies have shown that Asaia sp. bacteria can influence the larval development of some species of Anopheles, and the addition of this bacteria can accelerate the larval development. Anopheles stephensi larvae treated with the antibiotic rifampicin showed a delay in development and asynchrony in the appearance of posterior instars, since this antibiotic acts in the colonization and development of Asaia sp. [16, 17]. In another study, the genera Enterobacter and Serratia were seen to influence the digestive process due to hemolytic activity; when females of Ae. aegypti were treated with antibiotics (carbenicillin, tetracycline, spectinomycin, gentamicin, and kanamycin), the blood digestion was delayed. There was a change in the lysis of the red blood cells, which consequently reduced the egg production [18].

Regarding the influence of the intestinal microbiota on the biology of insects, one aspect that stands out is the ability of the microbial community to modulate infections caused by invading organisms, including pathogens transmitted by vectors to vertebrates $[19,20]$. It has been shown that after treatment with antibiotics (gentamicin + penicillin-streptomycin), Ae. aegypti and An. gambiae were more susceptible to dengue virus (DENV) and Plasmodium, respectively [21, 22]. Villegas et al. [23] demonstrated that the families Rhodobacteraceae and Desulfuromonadaceae could be considered as biomarkers for ZIKV infection. The reintroduction of the genera Paenibacillus, Proteus, and Chromobacterium in Ae. aegypti treated with penicillin-streptomycin led to a reduction in DENV infection, suggesting that metabolites secreted by bacteria belonging to native microbiota trigger basal immune activity capable of acting against DENV infection [24, 25]. On the other hand, intestinal colonization by Aeromonas sp., Escherichia coli, and Serratia marcescens was associated with increased susceptibility to DENV and/or CHIKV due to the suppression 
of the immune response of Ae. aegypti [26-28]. Intestinal bacteria can also interact directly with the pathogen, for example, through the production of antimicrobial toxins, generation of free radicals, or physical blocking of receptors in the intestinal epithelium through the formation of biofilm $[19,29]$.

These advances in the knowledge of interactions involving microbiota, pathogen, and vector have stimulated the search for new tools to control insects and the incidence of diseases, such as arboviruses. As pathogens and bacteria share the intestinal environment, the innate ability of some microorganisms to interfere with the susceptibility of the vector could be exploited to reduce the levels of transmission of arboviruses (DENV, ZIKV, and CHIKV) and Plasmodium (malaria) [24, 25, 29, 30]. This study describes the culture-dependent native microbiota associated with the female Ae. aegypti (strain PP-Campos). Using the cultivable resident isolates in the co-infection, we explore their possible influence or interference in establishing ZIKV infection and transmission.

\section{Methods}

\section{Aedes aegypti colony}

A well-established (since 2001) Brazilian closed colony of Ae. aegypti (strain PP-Campos), maintained at the Laboratory of Medical Entomology, Fiocruz, Minas Gerais, Brazil, was used in this study. Mosquitoes were reared and maintained under standard insectary conditions $\left(27^{\circ} \mathrm{C}, 80 \%\right.$ relative humidity, 12 -h light/12-h dark photoperiod) [23].

\section{Study groups}

Female 3-5-day-old Ae. aegypti mosquitoes were distributed into two experimental groups as follows:

I. Survey of cultivable bacteria; sucrose group: only fed on sucrose, i.e., non-blood-fed (UF); blood-fed group: (i) fed with non-infected blood (BF); (ii) fed with blood infected with ZIKV (BZIKV); (iii) pretreated with penicillin/streptomycin (pen/strep), and fed with non-infected blood (TBF); (iv) pretreated with pen/strep and fed with blood infected with ZIKV, i.e., gravid with developed ovaries, (TGZIKV).

II. Experimental co-infections: bacteria genera isolated from the group fed on sucrose, i.e., nonblood-fed (UF).

\section{Antibiotic pretreatment}

Before infective feeding, the mosquitoes were treated for three consecutive days with a $10 \%$ sterile sucrose solution containing $50 \mathrm{U} / \mathrm{ml}$ penicillin and $50 \mu \mathrm{g} / \mathrm{ml}$ streptomycin (pen/strep) ad libitum to remove or clear part of the native microbiota. The other groups were maintained for the same period in a $10 \%$ sterile sucrose solution. In all, about 200 mosquitoes were used in each group.

\section{Zika virus strain}

Brazilian human isolate of ZIKV from the state of São Paulo, the strain ZIKV/H. sapiens/Brazil/SPH/2015, was used in all the experiments [31]. The stocks were propagated in an Aedes albopictus cell line (C6/36) and grown in Leibovitz L-15 medium supplemented with $2 \%$ inactivated fetal bovine serum, $20 \mu \mathrm{g} / \mathrm{ml}$ gentamicin, $5 \mu \mathrm{g} /$ $\mathrm{ml}$ amphotericin B, and $200 \mathrm{U} / \mathrm{ml}$ penicillin [32]. Virus titration followed the $50 \%$ tissue culture infectious dose method [33].

\section{Experimental infection}

For 3 days before the infective meal, 3-5-day-old female Ae. aegypti mosquitoes were pretreated with $50 \mathrm{U} / \mathrm{ml}$ penicillin plus $50 \mu \mathrm{g} / \mathrm{ml}$ streptomycin in the sucrose meal (10\% ad libitum sucrose solution). The mosquitoes were infected via a membrane-feeding assay using a glass-feeding device that was filled with $400 \mu \mathrm{l}$ of mouse blood with or without $1 / 3$ of ZIKV (a titer of $1 \times 10^{5}$ plaque-forming units $(\mathrm{PFU}) / \mathrm{ml})$. The mosquitoes were allowed to feed for $1-2 \mathrm{~h}$ on the blood meal. After feeding, approximately 200 fully engorged females were separated into new cages and maintained on $10 \%$ sucrose solution ad libitum for up to 2,7 , and 14 days post-infection (dpi). Using realtime polymerase chain reaction ( $\mathrm{qPCR}$ ), as described below, $100 \%$ infection was determined on the seventh dpi in the groups used to survey cultivable bacteria.

\section{Bacterial diversity profiling using a culture-dependent technique}

The mosquitoes were first immobilized at $-20{ }^{\circ} \mathrm{C}$ for a few seconds and their surfaces sterilized by a 1-min submersion in $1 \%$ hypochlorite and $15-30 \mathrm{~s}$ in $70 \%$ ethanol, and then rinsed three times with phosphate-buffered saline (PBS) [34]. Subsequently, a pool of 10 midguts from each group was dissected under laminar flow, and bacterial members of the microbiota within each pool were isolated in tubes containing $200 \mu \mathrm{l}$ of brain-heart infusion broth (BHI) (Sigma, St. Louis, MO, USA), which is a non-selective medium for promoting the growth of an ample range of bacteria. The pools were gently macerated using a microtube homogenizer system. A subsample of the homogenate $(100 \mu \mathrm{l})$ was then pour-plated in the BHI agar medium at $27^{\circ} \mathrm{C}$ for $48 \mathrm{~h}$. The observed colonies, differentiated by color and morphological characteristics, were divided according to color, elevation, and shape, and were subjected to the spread plate technique in agar medium three times. The pure colonies 
expanded in the liquid medium, and each of the isolates, were differentiated by Gram staining and taxonomic profile. Finally, after the in vitro assays, the bacteria were re-plated and re-sequenced to ensure that the bacterial genera remained the same.

\section{S rRNA-oriented profiling}

A total of 52 bacterial isolates were obtained and the bacterial genomic DNA (gDNA) extraction was performed with the DNeasy Blood \& Tissue kit (Qiagen, Hilden, Germany) according to the manufacturer's instructions. The genomic material was quantified and its purity assessed using a spectrophotometer (NanoDrop, Thermo Fisher Scientific). Bacterial gDNA from each sample served as a template for a PCR assay using Illustra PuReTaq Ready-To-Go PCR beads (GE Healthcare, Buckinghamshire, UK) and the primers $16 \mathrm{~S}$ ribosomal RNA 27 sense 5'-AGAGTTTGATCA/CTGGCTCAG$3^{\prime}$, and 1492 antisense $5^{\prime}$-TACGGT/CTACCTTGTTAC GACTT- $3^{\prime}$. Amplification conditions were a $96{ }^{\circ} \mathrm{C}$ hold for $2 \mathrm{~min}, 30$ cycles of $95^{\circ} \mathrm{C}$ for $1 \mathrm{~min}, 50{ }^{\circ} \mathrm{C}$ for $1 \mathrm{~min}$, and $72{ }^{\circ} \mathrm{C}$ for $3 \mathrm{~min}$, followed by $5 \mathrm{~min}$ at $72{ }^{\circ} \mathrm{C}$. Amplified products were visualized on a $1 \%$ agarose gel (Fisher Bioreagents, NH, USA) and cleaned using the Wizard SV Gel and PCR Clean-up System (Promega, WI, USA). Twenty nanograms of the purified PCR product was sequenced using the PCR primers described above and the DYEnamic ET Terminator on a DNA sequencer (ABI 3730, Life Technologies, CA, USA). Sequences were aligned, merged into contigs, and trimmed using Sequencer software (version 5.4.6). Resulting sequences were analyzed for similarity using the sequence analysis tool RDP (Ribosomal Database Project- Update 5) and checked against the NR database (Non-redundant, NCBI database) using the BLASTN algorithm with default parameters [35]. The best BLAST hit was selected considering a $97 \%$ identity threshold. Taxonomic profiling of bacterial communities using $16 \mathrm{~S}$ rRNA sequences as a target has some well-known limitations, including database bias (e.g., due to a small amount of mosquito associated sequences deposited). The differences in each of the 16S rRNA variable regions are exhibited when resolving taxonomic levels [36, 37], and even cross-kingdom amplification [38], amongst other issues [39, 40]. For caution, we chose to report taxonomic identifications at the genus level only.

\section{Co-infection: bacterial isolates and ZIKV}

The bacterial genera isolated from the group fed on sucrose (UF) were used in the co-infection. As an "out-group," we used Lysinibacillus isolated from the midgut of Lutzomyia longipalpis. The females were previously treated with pen/strep as described above, and experimentally co-infected with ZIKV and $1 \times 10^{8}$ colony-forming units (CFU)/ml of the bacterial isolates. As a control group, untreated mosquitoes and mosquitoes pretreated with pen/strep were used and evaluated until the 14th dpi.

\section{Real-time qPCR for ZIKV detection and quantification}

Entire mosquito bodies and head-SGs were dissected from the experimental groups at 2, 7, and $14 \mathrm{dpi}$. These mosquito tissues were macerated and processed separately for RNA extraction (QIAamp Viral RNA Mini Kit, Qiagen, Hilden, Germany). Specific ZIKV primer and probe sets for were designed, as previously described by Lanciotti [41]. Primers were synthesized by Integrated DNA Technologies and probes with 5-FAM used as the reporter dye (Thermo Fisher). The number of viral copies was determined by automatic comparison with the specific set of standard samples. All real-time assays were performed using the TaqMan RNA-to-Ct 1-Step Kit, with amplification in the 7500 Fast and 7500 real-time PCR system, according to the manufacturer's protocol.

\section{Intrathoracic inoculation of infected salivary glands}

In parallel with the co-infection experiments, 14 days after the ZIKV-infective blood meal, 10 Ae. aegypti mosquitoes from each co-infective group were quickly killed by cold exposure. The salivary glands (SGs) were dissected, and the SGs ground with pestle tips in $10 \mu \mathrm{l}$ of L15 media (without antibiotics). Subsequently, the homogenates were used for the intrathoracic inoculation of 10 naïve 3-5-day-old Ae. aegypti (Nanoinject II, Drummond Scientific Co., Broomal, PA, USA). Postinoculation, these mosquitoes were maintained on $10 \%$ sucrose solution ad libitum for 7 days and processed via qPCR for ZIKV quantification [42].

\section{Saliva collection and detection of ZIKV}

The saliva from individual mosquitoes, co-infected through membrane feeding, were collected at 14 days after infection with ZIKV and the bacteria. In order to verify the effect of the bacterial isolates directly in the virus, Ae. aegypti were anesthetized with $\mathrm{CO}_{2}$ and kept on an ice plate while the wings and legs were removed. The proboscis of each mosquito was inserted into a $10 \mu \mathrm{l}$ pipette tip containing a 1:1 solution of $5 \mu \mathrm{l}$ sterile fetal bovine serum and 30\% sucrose solution. After $1 \mathrm{~h}$, the contents of the tips were collected in $1.5 \mathrm{ml}$ tubes and stored at $-70{ }^{\circ} \mathrm{C}$ until processing. ZIKV in mosquito saliva was quantified using qPCR [43].

\section{Statistical analysis}

The results were analyzed using the GraphPad Prism 7 program. The non-parametric Kruskal-Wallis 
Table 1 Genera of bacteria isolated from different physiological conditions of Ae. Aegypti

\begin{tabular}{|c|c|c|c|c|c|}
\hline Genus (Gram) & UF & $\mathrm{BF}$ & BZIKV & TBF & TGZIKV \\
\hline Acinetobacter (-) & & $x$ & & & \\
\hline Aeromonas (-) & $x$ & & & & \\
\hline Cedecea (-) & & $x$ & & & \\
\hline Cellulosimicrobium (+) & & & $x$ & & \\
\hline Elizabethkingia (-) & $x$ & & $x$ & $x$ & \\
\hline Enterobacter (-) & $x$ & $x$ & $x$ & $x$ & $x$ \\
\hline Lysinibacillus (+) & $x$ & & & & \\
\hline Pantoea (-) & & $x$ & & & \\
\hline Pseudomonas (-) & & & $x$ & $x$ & \\
\hline Serratia (-) & $x$ & & & & \\
\hline Staphylococcus (+) & & & & $x$ & $x$ \\
\hline
\end{tabular}

(+) Gram-positive and (-) Gram-negative. Groups: UF: fed on sucrose, nonblood-fed; BF fed with non-infected; BZIKV: fed with blood infected with ZIKV; TBF: pretreated with pen/strep and fed with non-infected blood; TGZIKV: pretreated and fed with blood infected with ZIKV, i.e., gravid with developed ovaries

test (ANOVA) was used for statistical analysis. Values of $P<0.05$ were considered significant $\left({ }^{*} P<0.05\right.$; ${ }^{* *} P<0.001 ;{ }^{* * * *} P<0.0001$; or $\left.{ }^{* * * *} P<0.00001\right)$.

\section{Results}

\section{Cultivable native microbiota of Ae. aegypti}

In 2018, Villegas et al. [23] revealed aspects of the profile of the native bacterial community of Ae. aegypti (strain PP-Campos) using $16 \mathrm{~S}$ amplicon sequencing (culture-independent method). Using the cultivable method and the same mosquito colony and the ZIKV strain described by Villegas, our results revealed 11 isolates (Acinetobacter, Aeromonas, Cedecea, Cellulosimicrobium, Elizabethkingia, Enterobacter, Lysinibacillus, Pantoea, Pseudomonas, Serratia, Staphylococcus). General aspects of the colonies presented a white color, circular shape, entire margin, and convex elevation, and eight were Gram-negative (72.7\%) and three were Gram-positive (27.3\%) (Table 1).

The analysis of the groups above allowed us to observe the following distribution of bacterial isolates: (1) Enterobacter present in all evaluated groups (UF, BF, BZIKV, TBF, and TGZIKV); (2) Elizabethkingia present in groups UF, BZIKV, and TBF; Pseudomonas present in groups BZIKV and TBF; and Staphylococcus in groups TBF and TGZIKV. The unique (exclusive) bacteria genera that were present in only one group were Aeromonas, Lysinibacillus, and Serratia (UF); Cedacea, Pantoea, and Acinetobacter (BF); and Cellulosimicrobium (ZIKV) (Fig. 1).

\section{Effect of bacterial isolates in ZIKV infection}

Based on the isolates Aeromonas, Elizabethkingia, Enterobacter, Lysinibacillus, and Serratia revealed in the UF group, three isolates were selected according to their effect, as follows: Aeromonas, associated with Ae. aegypti, which increased susceptibility to infection by DENV-2 [28]; Lysinibacillus, whose species Lysinibacillus sphaericus produces a toxin used as a larvicide in the control of Culex and Anopheles spp. [44]; Serratia, which is described as having anti-Plasmodium activity by stimulating the activation of the Toll receptor pathway in $A n$. stephensi [45] and in Ae. aegypti increases its susceptibility to DENV infection [46].

An isolate from another insect vector out-group Lysinibacillus-Lu. longipalpis was used due to its modulation in the course of infection by Leishmania [47], and this genus was found in our mosquito. We also evaluated whether these isolates were able to positively or negatively modulate ZIKV infection.

Whole insects were co-infected with four isolates and analyzed 2, 7, and $14 \mathrm{dpi}$. Note that there was still the presence of blood on the second day; however, Aeromonas and Lysinibacillus from Lu. Longipalpis and Serratia showed a twofold median reduction. This reduction in viral abundance may result in the completion of nutrients (bacteria and viruses) in the blood meal. At $7 \mathrm{dpi}$, when the digestive process was finished, we observed intense viral colonization, with a median of around $10^{7}$, and at 14 days, we observed a reduction in viral abundance. We conclude that it is difficult to determine any bacterial action concerning the virus using the entire insect.

The mosquitoes were simultaneously co-infected with bacteria and ZIKV, which was mixed into normal blood. On the second dpi, it was possible to observe a significant difference in the number of viral copies per mosquito between the control groups (treated with antibiotics and untreated) and the groups co-infected by ZIKV/Aeromonas and ZIKV/Lysinibacillus-Lu. longipalpis (out-group), On the seventh day, we observed the maintenance of the statistical difference between the control group and the group co-infected with ZIKV/Lysinibacillus-Lu. longipalpis. We also observed a difference between the control group that was treated and the group that was co-infected with ZIKV/Serratia. On the last day (14 dpi), we did not observe any differences in the intensity of the infection (Fig. 2).

The infection rate on the second dpi for the control groups (treated and untreated) and the group co-infected with ZIKV/Lysinibacillus-Ae. aegypti was $100 \%$. For ZIKV/Aeromonas, it was 90\%; for ZIKV/LysinibacillusLu. longipalpis, it was $80 \%$; and for ZIKV/Serratia, it was $60 \%$. On the seventh $\mathrm{dpi}$, the infection rate for the control groups (treated and untreated) and the co-infected 


\section{Cedacea}

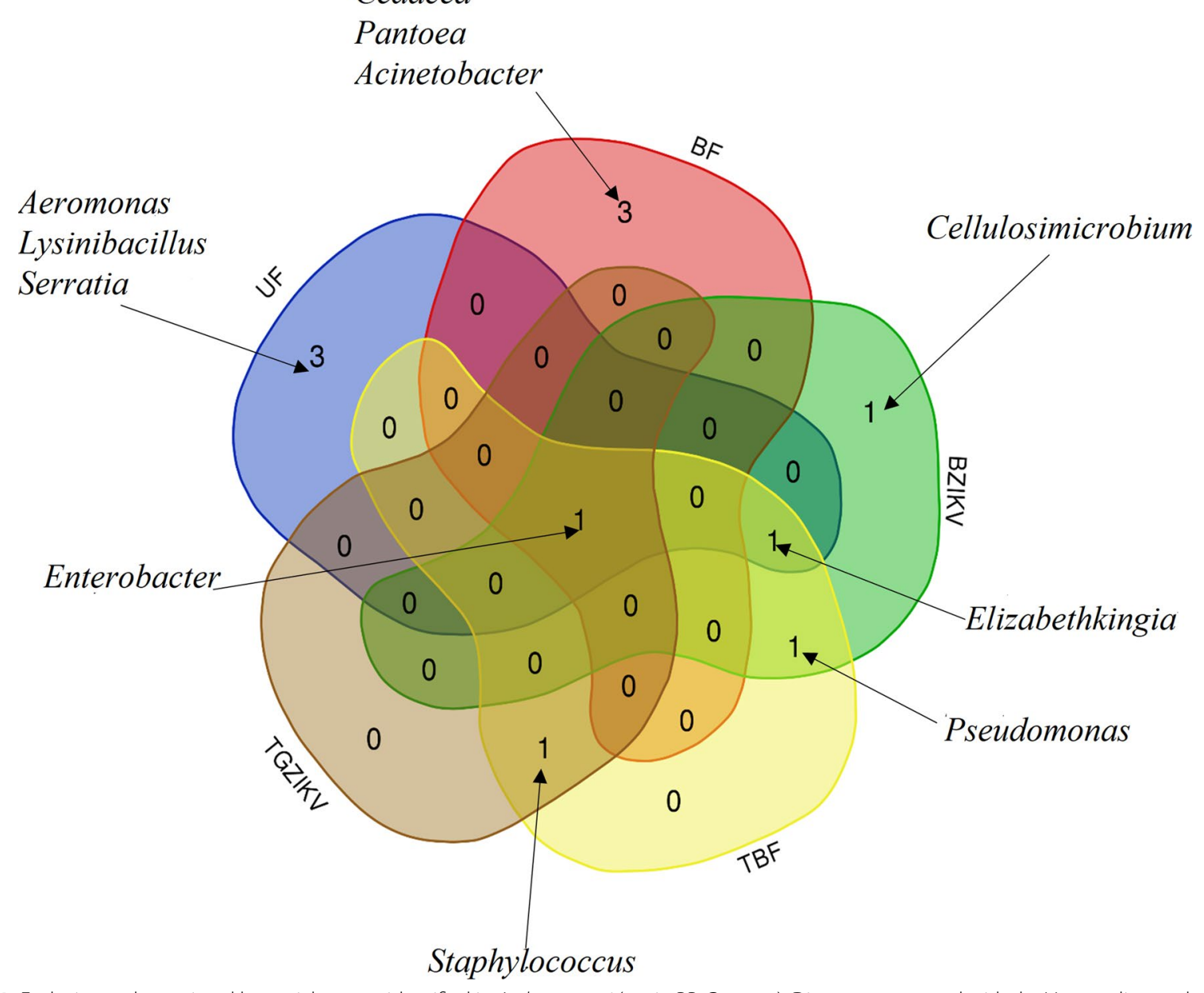

Fig. 1 Exclusive and associated bacterial genera identified in Aedes aegypti (strain PP-Campos). Diagram generated with the Venn online tool (http://bioinformatics.psb.ugent.be/cgibin/liste/Nenn/calculate_venn.htpl)

ZIKV/Serratia group was 100\%; for the ZIKV/Aeromonas and ZIKV/Lysinibacillus-Lu. longipalpis groups, it was 95\%; and for the co-infected ZIKV/Lysinibacillus-Ae. aegypti group, it was $90 \%$. On the 14th dpi, the infection rate for the control (treated) group was 100\%; for the coinfected ZIKV/Aeromonas, it was 96\%; for the control (untreated), co-infected ZIKV/Lysinibacillus-Lu. longipalpis and ZIKV/Serratia group, it was 90\%; and for the co-infected ZIKV/Lysinibacillus-Ae, aegypti group, it was $75 \%$ (Fig. 2).

In summary, the experimental co-infection assay was successful in all groups when analyzing the entire mosquito of co-infected mosquitoes at 2, 7, and $14 \mathrm{dpi}$. At the beginning of the infection (second dpi), we observed the smallest median numbers $\left(10^{2}\right.$ to $\left.10^{4}\right)$ in cDNA viral copies. Statistically significant differences were observed in mosquitoes co-infected with Aeromonas and Lysinibacillus-Lu. longipalpis ( ${ }^{* * *} P=0.001$, $\left.{ }^{* * *} P=0.0001\right)$. On the seventh day, after the digestive process, it was possible to note a higher number $\left(10^{7}\right)$ of copies in those co-infected with Lysinibacillus from Ae. aegypti, and Lu. longipalpis and Serratia (" $P=0.05, " * P=0.001)$. However, on the 14th day, we noticed a small reduction.

\section{Impact of co-infection in the body and head-SG}

No positive or negative changes in viral abundance were observed on the 14th dpi.

Since we did not observe any viral abundance using the whole insect, we performed a second infection using isolates identical to those described above and analyzed the body and head-SG. The samples were analyzed 14 days after the intrinsic incubation. We observed medians of around $10^{7}$ and a smaller number of copies in the head-SG. In summary, we can assume that after colonization of the body, there was a 


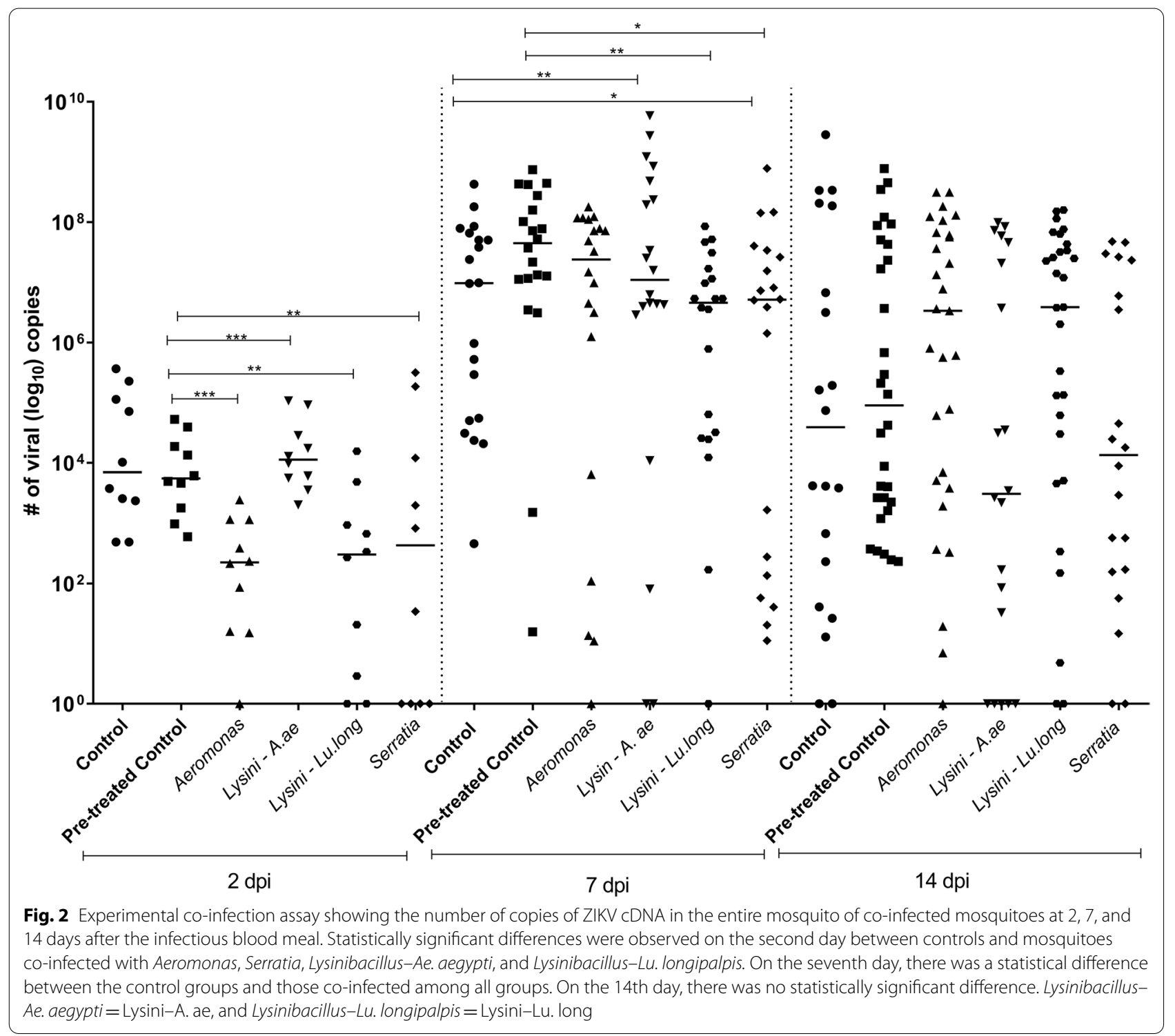

reduction in the invasion of the gland, perhaps indicating some effective barrier related to the infection of the salivary gland.

Therefore, we wondered whether there was any barrier or variation regarding the abundance of infection in the target organs of the mosquito. In all the groups, we observed greater viral abundance in the entire mosquito (Fig. 2).

In summary, the infection rate was $100 \%$ in the head-SG for the control group (treated) and for the co-infected ZIKV/Aeromonas. For the control group (untreated), the ZIKV/Lysinibacillus-Lu. longipalpis co-infected group, and the co-infected ZIKV/Serratia group, infection was $80 \%$, and for the co-infected ZIKV/Lysinibacillus-Ae. aegypti group, it was
$50 \%$. In the body, the infection rate for the control groups (treated and untreated) and the co-infected ZIKV/Lysinibacillus-Ae. aegypti, ZIKV/LysinibacillusLu. longipalpis and ZIKV/Serratia groups was 100\%, and for the co-infected ZIKV/Aeromonas group, it was 90\% (Fig. 3).

Interestingly, the group with the highest median number of viral copies in the head-SG was the out-group that was co-infected with Lysinibacillus-Lu. longipalpis, and the group with the lowest number of viral copies was the one co-infected with Lysinibacillus-Ae. aegypti (Fig. 3). Our results suggest that Lysinibacillus-Ae. aegypti positively influenced the reduction in viral load compared to the group co-infected with Lysinibacillus-Lu. longipalpis. 


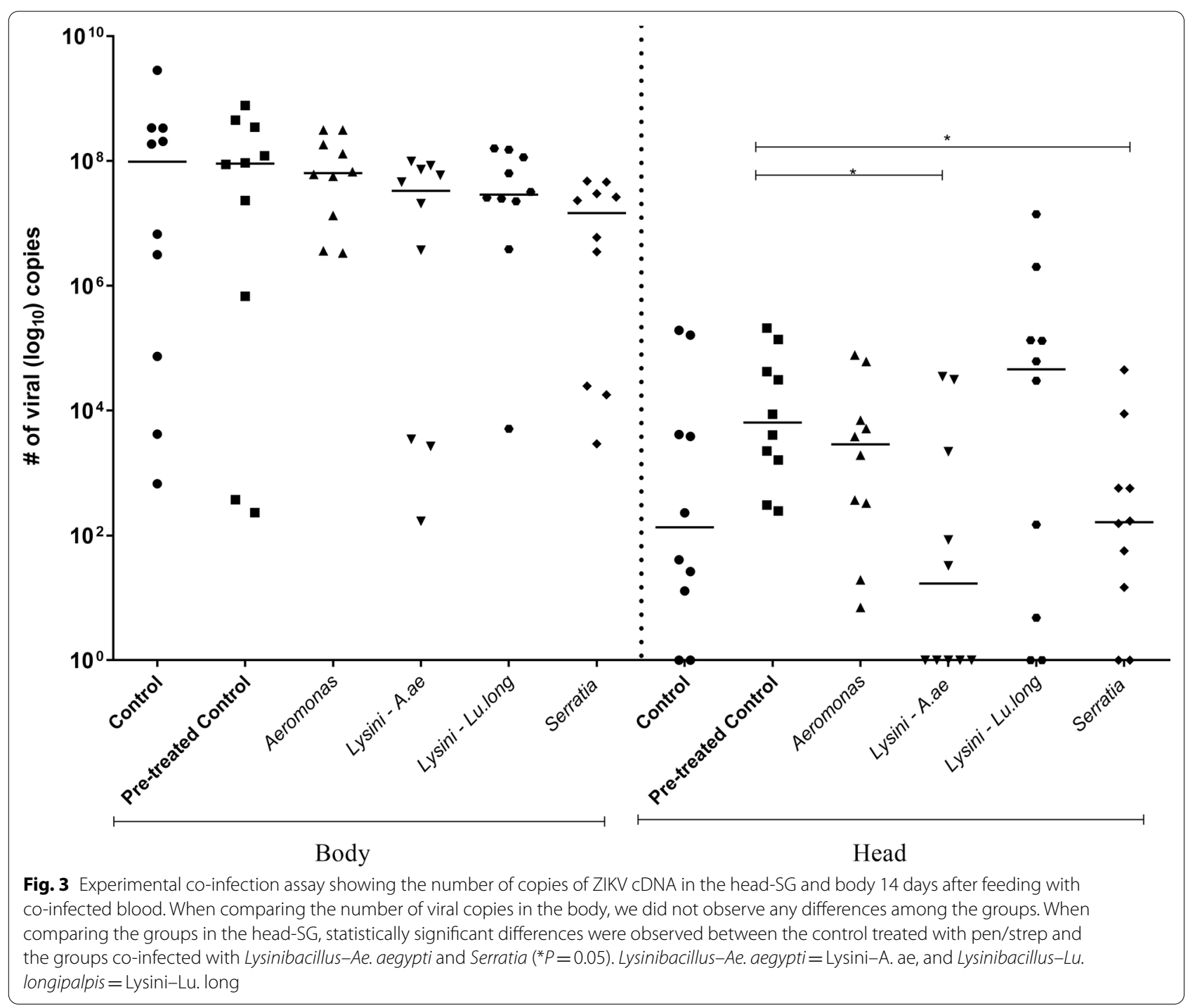

\section{Poof of viability of ZIKV after mosquito infection}

To assess the viability of ZIKV in co-infection (i.e., the capacity of the virus to reach the salivary gland and be transmitted), we analyzed the viral abundance on the 14th dpi (Fig. 3).

To avoid misinterpretation due to quantification of numbers of viral copies in the salivary glands (i.e., quantifying genetic material), a subset of mosquitoes from the second infection were used in the intrathoracic injection, and we analyzed whether the virus was able to establish a new infection.

The intrathoracic injection serves to avoid the midgut barrier and facilitate the infection, and the mosquitoes were analyzed at $7 \mathrm{dpi}$ and presented $30-50 \%$ infection using Aeromonas, Lysinibacillus, and Serratia and 100\% for Lu. longipalpis.
Additionally, the salivary glands of the mosquitoes were dissected, and the homogenate was inoculated into the thorax of naïve mosquitoes. At 7 days after the intrathoracic infection, it was possible to observe the viral load in these mosquitoes. The infection rate for the ZIKV/Lysinibacillus-Lu. longipalpis co-infected group was $100 \%$; for the control (treated) group, it was 80\%; for the co-infected ZIKV/Serratia, it was $70 \%$; and for the control groups (untreated), co-infected ZIKV/Aeromonas, and co-infected ZIKV/LysinibacillusAe. aegypti groups, it was $50 \%$.

The number of copies of ZIKV in the control group treated with antibiotics was higher than that in the control group (untreated) and was similar in the group coinfected with ZIKV/Lysinibacillus-Lu. longipalpis, with a median of around $10^{9}$ viral copies per mosquito. In the other groups co-infected with ZIKV/Aeromonas, 


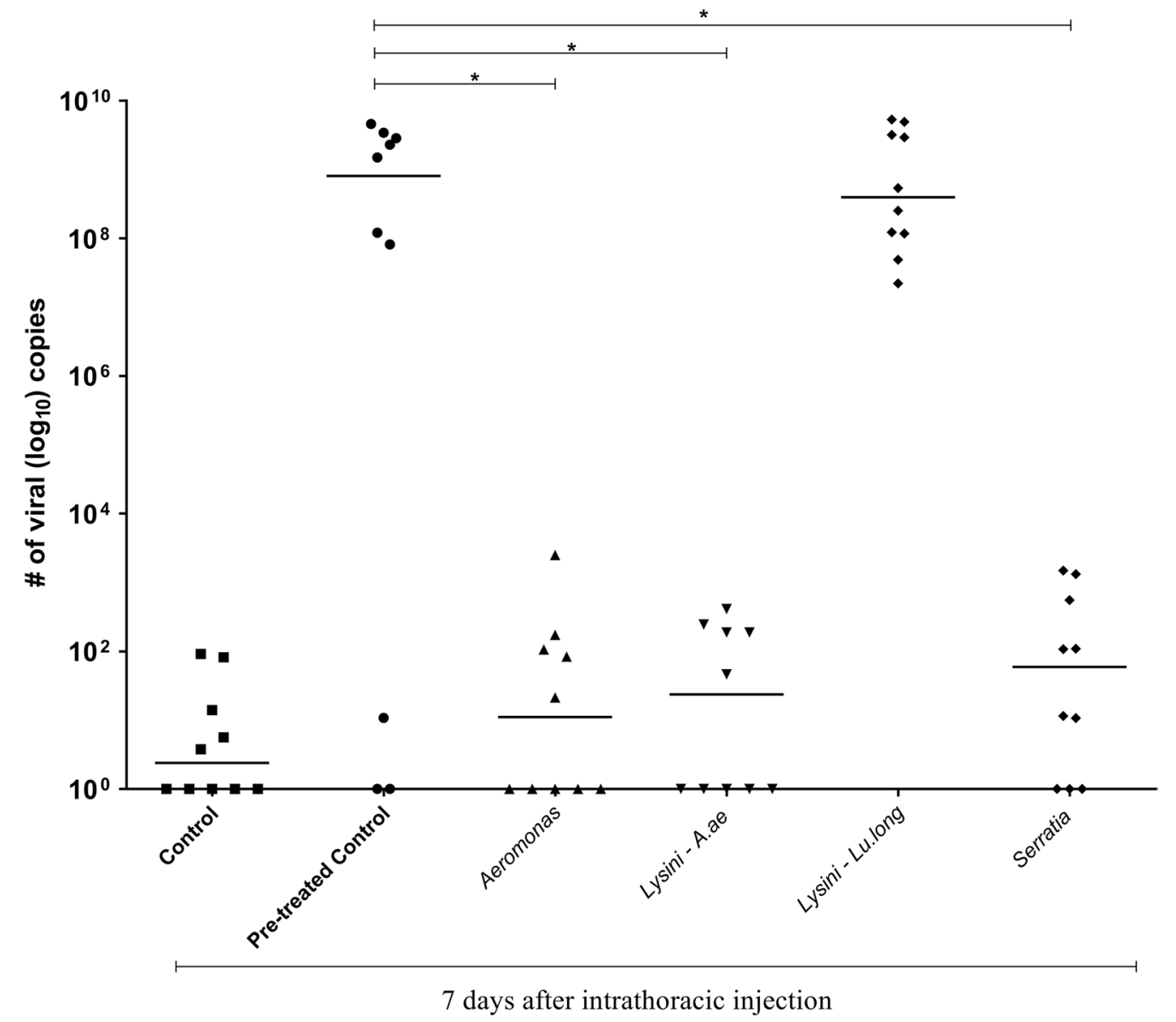

Fig. 4 Experimental assessment of the viability of ZIKV in co-infection. The infected salivary gland homogenates from mosquitoes analyzed for viral abundance on 14 dpi were inoculated by intrathoracic injection in naïve mosquitoes, and the viral load was analyzed 7 days after infection. Statistically significant differences were observed for most groups when comparing the number of viral copies of cDNA from co-infected mosquitoes with controls ( $\left.{ }^{*} P=0.05,{ }^{* * *} P=0.00001\right)$. Lysinibacillus-Ae. aegypti $=$ Lysini-A. ae, and Lysinibacillus-Lu. longipalpis $=$ Lysini-Lu. long

ZIKV/Lysinibacillus-Ae. aegypti, and ZIKV/Serratia, the number of copies of the ZIKV was approximately $10^{2}$ copies of cDNA per mosquito (Fig. 4). Our results show that in all the injected groups, the virus remained viable at $7 \mathrm{dpi}$.

\section{The influence of co-infection on transmission}

Concomitantly, to assess the impact of co-infection on transmission, the saliva of part of the mosquitoes that were infected was analyzed. Salivation revealed a median of around $10^{4}$ copies in the control group pretreated with pen/strep and around $10^{3}$ in the group co-infected with ZIKV/Aeromonas. It was not possible to detect ZIKV in the other groups analyzed (Fig. 5). In summary, the virus was not detected by $\mathrm{qPCR}$ in saliva, which could have an impact on transmission-i.e., mosquitoes co-infected with ZIKV/Lysinibacillus-Ae. aegypti, ZIKV/Lysinibacillus-Lu. longipalpis cannot transmit viable viruses-and in ZIKV/Serratia, only one was positive, which implies that Serratia may also interfere in transmission, even though viruses in the head were detected, as shown in Fig. 3, which influences vector competence.

\section{Discussion}

Zika fever is an emerging systemic arboviral disease caused by ZIKV. Vector transmission, more specifically mosquitoes of the genus Aedes, is the virus's primary mode of transmission to the host $[48,49]$. The first autochthonous case of ZIKV infection in the Americas was reported in northeastern Brazil in May 2015. Subsequently, Zika spread rapidly throughout Brazil, reaching its peak in 2016 with 273,904 reported cases [50-52]. The transmission of ZIKV persists, although the number of cases has decreased since 2018 (19,020 reported cases) [51]. Areas with competent vectors are at risk of 


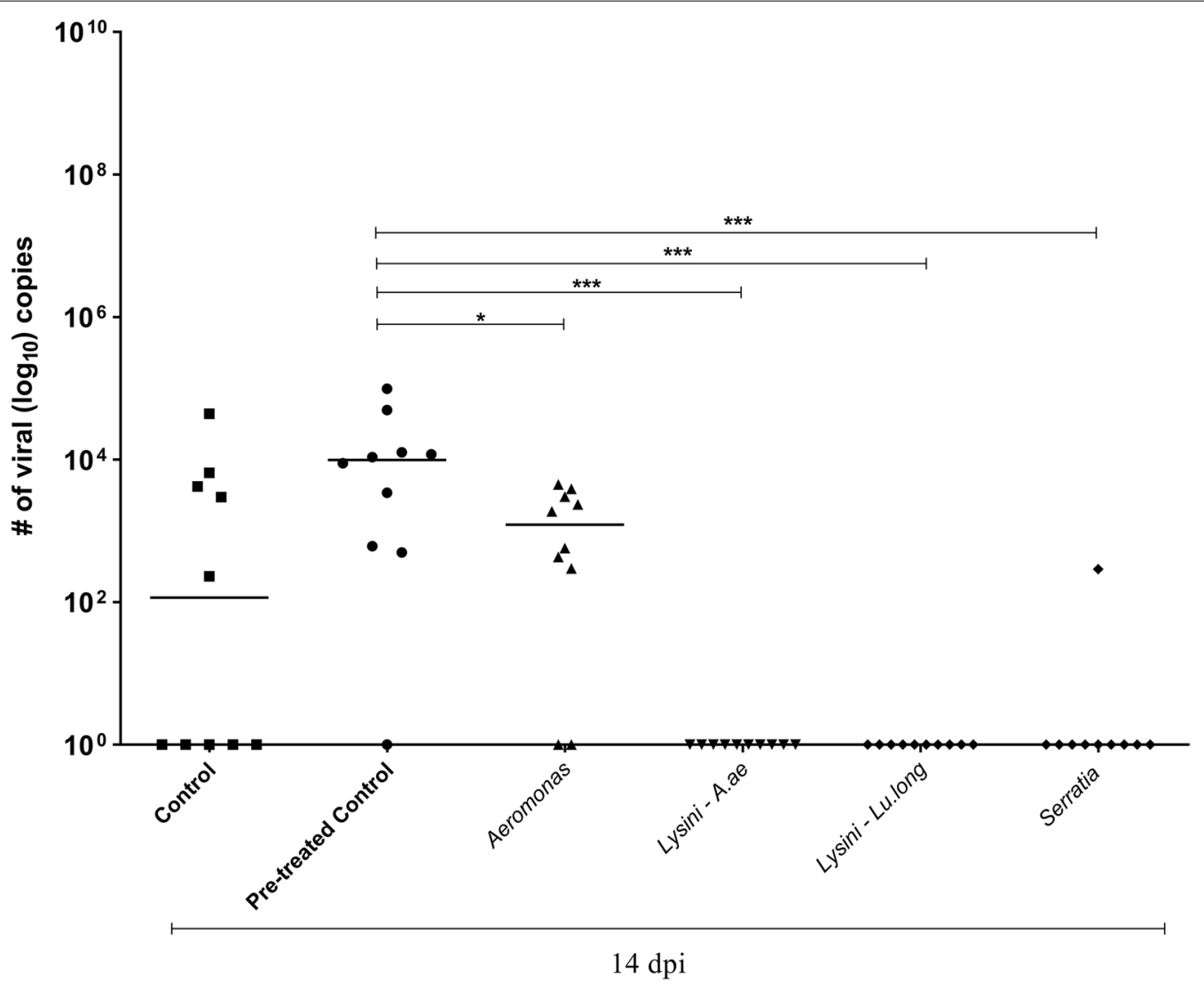

Fig. 5 Number of ZIKV cDNA copies in the saliva of mosquitoes infected 14 days after feeding with co-infected blood. Statistically significant differences were observed for all groups when comparing the number of viral copies of CDNA from the co-infected mosquito saliva with the treated control $\left({ }^{*} P=0.05,{ }^{* * *} P=0.0001\right)$. Against the untreated control, statistically significant differences were observed in the co-infection with Lysinibacillus, isolated from Aedes aegypti and Lu. Iongipalpis ( ${ }^{*} P=0.05$ ). Lysinibacillus-Ae. aegypti $=$ Lysini-A. ae, and Lysinibacillus-Lu. longipalpis $=$ Lysini-Lu. long

re-emergence of ZIKV, making advances in the search for new strategies for vector control essential. Knowledge regarding the microbiota associated with mosquitoes of the genus Aedes is fundamental in order to understand the existing interaction with the pathogen and elucidate its impact on vector competence.

Mosquitoes host a diverse community of microorganisms that can influence development [15], reproduction [18], and susceptibility to pathogens [53]. In this study, using a cultivable bacteria method, we identified the microbiota resident in Ae. aegypti (Cepa PP-Campos) under distinct feeding aspects. We isolated 11 bacterial genera, of which eight were Gram-negative (72.7\%) (Acinetobacter, Aeromonas, Cedecea, Elizabethkingia, Enterobacter, Pantoea, Pseudomonas, and Serratia) and three were Gram-positive (27.3\%) (Cellulosimicrobium, Lysinibacillus, and Staphylococcus), and these were distributed in five experimental groups (UF, BF, BZIKV, TBF, and TGZIKV). These findings are in agreement with those of Villegas et al. [23] who, through the use of high-throughput sequencing, found Gram-negative bacteria to be predominant in mosquitoes fed sucrose and/ or blood and infected with ZIKV.

The bacterial diversity showed a change according to the distinct feeding aspects evaluated. As shown in our results (Table 1), our analysis among the experimental groups revealed four genera (Enterobacter, Elizabethkingia, Pseudomonas, and Staphylococcus) associated between the groups. Enterobacter was identified in all experimental groups (UF, BF, BZIKV, TBF, and TGZIKV). In addition, it is already considered the prevalent genus of intestinal bacteria cultivable in Ae. aegypti and Ae. albopictus [54], and other studies have described its ability to deal with oxidative stress caused by feeding with blood [55] and its association in the blood digestion process $[18,56]$. Elizabethkingia, identified in the UF, BZIKV, and TBF groups, has also been described in Ae. aegypti fed with sucrose [57] and in the groups fed with sucrose, fed with blood, fed with blood infected with $\mathrm{ZIKV}$, and gravid mosquitoes infected with ZIKV that 
were described by Villegas et al. [23], and represented at the family level (Flavobacteriaceae). Pseudomonas, identified in the BZIKV and TBF groups, are present in Ae. aegypti evaluated according to different food sources [23, 53, 58, 59]. Staphylococcus was identified in the TBF and TGZIKV groups. Yadav et al. [54, 60] isolated the Staphylococcus genus from emerged Ae. aegypti mosquitoes [51] and Ae. albopictus fed on blood. Additionally, the Staphylococcaceae family was described in the groups (fed with sucrose, fed with blood, fed with blood infected with ZIKV, and gravids infected with ZIKV) that were evaluated by Villegas et al. [23].

Some bacterial isolates were unique to only one experimental group, such as Aeromonas, Lysinibacillus, and Serratia, which were present in the UF group. Aeromonas has been described in Ae. aegypti [24], Ae. albopictus [60], Culex quinquefasciatus [61], and An. gambiae [62]. The genus Lysinibacillus was isolated from emerged Ae. aegypti and Ae. albopictus adults [54] and pupa and adults of Lu. longipalpis [47]. Serratia was isolated from Ae. aegypti by Apte-Deshpande et al. [27], who identified the species Serratia odorifera in the midgut of larvae and females of colonized mosquitoes.

Cedecea, Pantoea, and Acinetobacter were identified only in the BF group. Gusmão et al. [56] also isolated Cedecea from the midgut of Ae. aegypti fed on blood (culture-independent method). Acinetobacter was shown to be frequently associated with different species of mosquitoes, including Ae. aegypti, Ae. albopictus, Ae. triseriatus, An. stephensi, Cx. pipiens, Cx. quinquefasciatus, and Psorophora columbiae [63]. One study, carried out by Minard et al. [64], demonstrated that in Ae. albopictus, the species Acinetobacter baumannii and Acinetobacter johnsonii may be involved in the blood digestion process. Pantoea was identified in a metagenomic analysis of the species Ae. aegypti [23] and Cx. quinquefasciatus [59]. The genus Cellulosimicrobium was isolated only in the BZIKV group. This genus was recently described by Schuman et al. [65], and was isolated from the intestine of the termite Mastotermes darwiniensis [66] and Ae. albopictus [67].

Part of the microbiota in adult mosquitoes acquired from the larval habitat and another fraction is dependent on food resources, whether they are fed sucrose or blood (in the case of females), and this significantly affects the diversity and abundance of the bacterial population [68]. The factors that may be related to bacterial diversity in the experimental groups in this study are the interactions between microbial communities and treatment with antibiotics. For example, Serratia and Cedecea spp. demonstrated co-exclusion relationships with dominant taxa, such as members of the genera Asaia, Pseudomonas, and Enterobacter in the microbiota of wild and colonized Ae aegypti, Ae. albopictus, and Cx. quinquefasciatus [69]. Regarding antibiotic treatment, Gaio et al. [18] demonstrated that treatment of Ae. aegypti with antibiotics (carbenicillin, tetracycline, spectinomycin, gentamicin, and kanamycin) promoted a reduction in the abundance of cultivable bacteria; Enterobacter sp. and Serratia sp. were the predominant bacteria and were associated with hemolytic activity [18]. No reduction in abundance was observed; however, the antibiotic treatment did reveal the presence of Enterobacter, Elizabethkingia, Pseudomonas, and Staphylococcus [18].

To demonstrate the bacterial diversity described for Aedes spp. mosquitoes, we compared our results with the genera already identified in the current literature. We sought to demonstrate how the native microbiota could be modulated by the food source, and for this we compared the bacterial genera isolated in the unfed mosquitoes, mosquitoes fed with sucrose, blood, or blood infected with arbovirus (fed), and the isolated genera in this study (Additional file 1: Figure S1).

As shown in Additional file 1: Figure S1, the genera Aeromonas, Elizabethkingia, Enterobacter, Pantoea, Pseudomonas, and Serratia were identified in all established conditions. These findings suggest that, despite the modulation of the microbiota by the habitat and food source, there are bacteria that can adapt under the changing conditions of the holobiont $[15,23,63]$.

Regarding the interaction between the mosquito, the virus, and the microbiota, we performed a reintroduction of bacteria in the Ae. aegypti treated with the pen/ strep antibiotic in order to identify whether these bacteria could positively or negatively influence ZIKV infection. On the second day after feeding, a reduction in the intensity of infection was observed between the groups co-infected with ZIKV/Aeromonas and LysinibacillusLu. longipalpis compared to the control groups (treated and untreated). On the seventh day, the reduction in viral load in the ZIKV/Lysinibacillus-Ae. aegypti coinfected group persisted, and we also observed a difference between the control group (treated) and the group co-infected with ZIKV/Serratia. In contrast, previous studies have revealed an association between Serratia sp. and increased infectivity and prevalence of DENV, ZIKV, and CHIKV in Ae. aegypti $[26,27,46]$. Aeromonas has also been associated with increased susceptibility to Ae. aegypti infection by DENV-2 [28]. Ramirez et al. [24] evaluated the influence of the reintroduction of Proteus and Paenibacillus on DENV infection in Ae. aegypti, and a decrease in the viral load of DENV was observed. At the taxonomic level, Proteus belongs to the family Enterobacteriaceae, and Paenibacillus belongs to the phylum Firmicutes, correlating with the genera Serratia 
(Enterobacteriaceae) and Lysinibacillus (phylum Firmicutes) evaluated in this study.

When comparing body and head-SG on the 14th day after feeding, we observed a significant reduction in viral load between the groups evaluated. It is known that the mosquito-virus interaction process can be influenced by anatomical barriers, digestive enzymes, the immune system, and the microbiota [70,71]. The interaction between microbiota, immune system, and DENV in Ae. aegypti was demonstrated by Ramirez et al. [24]. It was observed that the reintroduction of isolated bacteria in the midgut of antibiotic-treated mosquitoes (pen/strep) caused changes in the abundance of antimicrobial peptide genes, including cecropins, gambicins, and attacins. The authors suggested that the modulation of the abundance of transcripts of the immunological gene by the reintroduced bacteria may have a nocuous effect on DENV infection [24].

Interestingly, the group with the highest number of viral copies in the head-SG was co-infected with the Lysinibacillus-Lu. longipalpis out-group, and the group with the lowest number of viral copies was the one coinfected with Lysinibacillus-Ae. aegypti. One possible explanation for this finding is the interaction between the insect, species vectors, and native microbiota. When $L u$. longipalpis was co-infected with Lysinibacillus and Leishmania spp., a significant reduction in the parasitic load was observed [47]. By simultaneously analyzing viral abundance on the 14th dpi, we sought to assess the viability of ZIKV in co-infection via inoculation of infected SGs in the thorax of naive mosquitoes. On the seventh dpi, it was possible to observe viable viruses in all the groups evaluated, with a median of around $10^{9}$ viral copies per mosquito. Secundino et al. [43] reported intrathoracic-inoculated Ae. aegypti (PP-Campos) with SGs infected with ZIKV viable viruses after 14 days, with the amount ranging from $2.7 \times 10^{6}$ to $5.4 \times 10^{7}$, and with a median of $1.49 \times 10^{7}$ copies of ZIKV cDNA.

To demonstrate the impact of co-infection on transmission, the saliva of part of the infected mosquitoes (via the membrane) was analyzed. Salivation revealed a median of around $10^{4}$ copies in the control group pretreated with pen/strep, and around $10^{3}$ in the group co-infected with Aeromonas. Depending on the virus, mosquito species, and the quantification method employed, the estimated level of virus inoculated in mosquito saliva ranges from approximately $10^{1}$ to $10^{7} \mathrm{PFU}[72,73]$. Consequently, mosquitoes co-infected with ZIKV/Lysinibacillus-Ae. aegypti, ZIKV/Lu. longipalpis did not present viable virus in their saliva, even though the virus was detected in the head-SG, as shown in Fig. 3. As demonstrated by other researchers, the detection of ZIKV in the saliva is a potential indicator of transmission to the host $[74,75]$.

\section{Conclusions}

In summary, we demonstrate that the physiological conditions assessed herein influenced the composition of bacterial diversity. In the co-infection, among ZIKV, Ae. aegypti and the bacterial isolates, the ZIKV/Lysinibacillus-Ae. aegypti group had the lowest number of viral copies in the head-SG, which means that it negatively affected the vectorial competence. However, when the saliva was analyzed after forced feeding, no virus was detected in the mosquito groups ZIKV/Lysinibacillus-Lu. longipalpis or Ae. aegypti, and was partially detected in only one mosquito (ZIKV/Serratia). These results indicate that the combinations do not allow the development of viable viruses and thus show that they hold important potential as a biological control tool.

\section{Abbreviations}

UF: Fed on sucrose, i.e., non-blood-fed; BF: Fed with non-infected blood; BZIKV: Fed with blood infected with ZIKV; TBF: Pretreated with pen/strep and fed with non-infected blood;TGZIKV: Pretreated with pen/strep and fed with blood infected with ZIKV, i.e., gravid with developed ovaries; dpi: Days post-infection.

\section{Supplementary Information}

The online version contains supplementary material available at https://doi. org/10.1186/s13071-022-05160-7.

Additional file 1: Figure S1. Bacterial diversity of Aedes spp. under different distinct feeding aspects [53, 56, 58, 59, 64, 68, 76-81]. Purple: unfed; green: fed (sucrose, blood, or blood infected with arbovirus); blue: isolated genera in this study. The Circos plot was generated using an online tool. http://circos.ca.

\section{Acknowledgements}

The following Brazilian agencies partially funded this study: Oswaldo Cruz Foundation (FIOCRUZ), Brazilian Council for Scientific and Technological Development (CNPq), and INCT Entomologia Molecular. NS and PP received PQ fellowships from CNPq. RM received a fellowship from FAPEAM as a Masters student. TBC received a fellowship from Capes as a PhD student. This study was partially supported by the Coordination for the Improvement of Higher Education Personnel (Coordenação de Aperfeiçoamento de Pessoal de Nível Superior-CAPES)_Finance Code 001.

\section{Authors' contributions}

Conceived and designed the experiments: NFCS, PFPP. Performed the experiments: RMN, TBC, JLFD, RSMG, BAC. Analyzed the data: NFCS, RMN, TBC, RSMG. Wrote the paper: NFCS, PFPP, RMN, TBC. All authors read and approved the final manuscript.

Availability of data and materials

The microbiota is a huge project still in analysis. Additional data is available from the corresponding author on reasonable request.

\section{Declarations}

Ethics approval and consent to participate

This study was conducted in conformity with the Oswaldo Cruz Foundation's Handbook for Animal Use (Ministry of Health of Brazil) and was approved by the Animal Use Ethics Committee at the Oswaldo Cruz Foundation (Approval number LW-17/15). 


\section{Consent for publication}

Not applicable.

\section{Competing interests}

The authors declare that they have no competing interests.

\begin{abstract}
Author details
${ }^{1}$ Laboratorio de Entomologia Médica, Instituto René Rachou-FIOCRUZ-Minas, Belo Horizonte, Minas Gerais, Brazil. ${ }^{2}$ Instituto de Pesquisas Clínicas Carlos Borborema, Fundação de Medicina Tropical Dr. Heitor Vieira Dourado, Manaus, Amazonas, Brazil. ${ }^{3}$ Programa de Pós-Graduação em Medicina Tropical, Universidade do Estado do Amazonas, Manaus, Brazil. ${ }^{4}$ Programa de Pós-Graduação em Ciências da Saúde, IRR-FIOCRUZ-Minas, Belo Horizonte, Minas Gerais, Brazil.
\end{abstract}

Received: 28 June 2021 Accepted: 10 January 2022

Published online: 17 February 2022

\section{References}

1. World Health Organization. Vector-borne diseases. 2020. https://www. who.int/news-room/fact-sheets/detail/vector-borne-diseases.

2. Maitra A, Cunha-Machado AS, de Souza Leandro A, Costa FM, Scarpassa VM. Exploring deeper genetic structures: Aedes aegypti in Brazil. Acta Trop. 2019;195:68-77.

3. Bowman LR, Donegan S, McCall PJ. Is Dengue vector control deficient in effectiveness or evidence?: systematic review and meta-analysis. PLoS Negl Trop Dis. 2016;10:e0004551.

4. World Health Organization. Dengue guidelines for diagnosis, treatment, prevention and control: new edition. Geneva: World Health Organization; 2009.

5. Abad-Franch F, Zamora-Perea E, Luz SLB. Mosquito-disseminated insecticide for citywide vector control and its potential to block arbovirus epidemics: entomological observations and modeling results from Amazonian Brazil. PLoS Med. 2017;14:e1002213.

6. Wang S, Jacobs-Lorena M. Paratransgenesis applications: fighting malaria with engineered mosquito symbiotic bacteria. In: Vector microbiome and innate immunity of arthropods. Elsevier Inc. 2017; 1:219-234.

7. Evans BR, Kotsakiozi P, Costa-da-Silva AL, Ioshino RS, Garziera L, Pedrosa MC, et al. Transgenic Aedes aegypti mosquitoes transfer genes into a natural population. Sci Rep. 2019;9:13047.

8. Scolari F, Casiraghi M, Bonizzoni MM. Aedes spp. and their microbiota: a review. Front Microbiol. 2019;10:2036.

9. Ricci I, Damiani C, Capone A, DeFreece C, Rossi P, Favia G. Mosquito/ microbiota interactions: from complex relationships to biotechnological perspectives. Curr Opin Microbiol. 2012;15:278-84.

10. Durvasula RV, Gumbs A, Panackal A, Kruglov O, Aksoy S, Merrifield RB, et al. Prevention of insect-borne disease: an approach using transgenic symbiotic bacteria. Proc Natl Acad Sci USA. 1997;94:3274-8.

11. Bongio NJ, Lampe DJ. Inhibition of Plasmodium berghei development in mosquitoes by effector proteins secreted from Asaia sp. bacteria using a novel native secretion signal. PLoS ONE. 2015;10:e0143541.

12. Shane JL, Grogan CL, Cwalina C, Lampe DJ. Blood meal-induced inhibition of vector-borne disease by transgenic microbiota. Nat Commun. 2018:9:4127.

13. Jupatanakul N, Sim S, Dimopoulos G. The insect microbiome modulates vector competence for arboviruses. Viruses. 2014;6:4294-313. https://doi. org/10.3390/v6114294.

14. Landmann F. The Wolbachia endosymbionts. Microbiol Spectr. 2019;7:7.2.25

15. Coon KL, Vogel KJ, Brown MR, Strand MR. Mosquitoes rely on their gut microbiota for development. Mol Ecol. 2014;23:2727-39.

16. Chouaia B, Rossi P, Epis S, Mosca M, Ricci I, Damiani C, et al. Delayed larval development in Anopheles mosquitoes deprived of Asaia bacterial symbionts. BMC Microbiol. 2012;12:S2

17. Mitraka E, Stathopoulos S, Siden-Kiamos I, Christophides GK, Louis C. Asaia accelerates larval development of Anopheles gambiae. Pathog Glob Health. 2013;107:305-11.

18. Gaio AdO, Gusmão DS, Santos AV, Berbert-Molina MA, Pimenta PF, Lemos FJ. Contribution of midgut bacteria to blood digestion and egg production in Aedes aegypti (diptera: culicidae) (L.). Parasites Vectors. 2011:4:105.

19. Azambuja P, Garcia ES, Ratcliffe NA. Gut microbiota and parasite transmission by insect vectors. Trends Parasitol. 2005;21:568-72.

20. Cirimotich CM, Ramirez JL, Dimopoulos G. Native microbiota shape insect vector competence for human pathogens. Cell Host Microbe. 2011;10:307-10.

21. Xi Z, Ramirez JL, Dimopoulos G. The Aedes aegypti toll pathway controls Dengue virus infection. PLOS Pathog. 2008;4:e1000098.

22. Dong Y, Manfredini F, Dimopoulos G. Implication of the mosquito midgut microbiota in the defense against malaria parasites. PLoS Pathog. 2009;5:e1000423.

23. Villegas LEM, Campolina TB, Barnabe NR, Orfano AS, Chaves BA, Norris $D E_{1}$ et al. Zika virus infection modulates the bacterial diversity associated with Aedes aegypti as revealed by metagenomic analysis. PLoS ONE. 2018;13:e0190352.

24. Ramirez JL, Souza-Neto J, Torres Cosme R, Rovira J, Ortiz A, Pascale JM, et al. Reciprocal tripartite interactions between the Aedes aegypti midgut microbiota, innate immune system and Dengue virus influences vector competence. PLoS Negl Trop Dis. 2012;6:e1561.

25. Ramirez JL, Short SM, Bahia AC, Saraiva RG, Dong Y, Kang S, et al. Chromobacterium Csp_P reduces malaria and dengue infection in vector mosquitoes and has entomopathogenic and in vitro anti-pathogen activities. PLoS Pathog. 2014;10:e1004398.

26. Apte-Deshpande AD, Paingankar MS, Gokhale MD, Deobagkar DN. Serratia odorifera mediated enhancement in susceptibility of Aedes aegypti for chikungunya virus. Indian J Med Res. 2014;139:762-8.

27. Apte-Deshpande A, Paingankar M, Gokhale MD, Deobagkar DN. Serratia odorifera a midgut inhabitant of Aedes aegypti mosquito enhances its susceptibility to Dengue-2 virus. PLoS ONE. 2012;7:e40401.

28. Mourya DT, Pidiyar V, Patole M, Gokhale MD, Shouche Y. Effect of midgut bacterial flora of Aedes aegypti on the susceptibility of mosquitoes to Dengue viruses. WHO Regional Office for South-East Asia, vol. 26. 2002. pp. 190-4

29. Cirimotich CM, Dong Y, Clayton AM, Sandiford SL, Souza-Neto JA, Mulenga $M$, et al. Natural microbe-mediated refractoriness to Plasmodium infection in Anopheles gambiae. Science. 2011;332:855-8.

30. Bahia AC, Dong Y, Blumberg BJ, Mlambo G, Tripathi A, BenMarzoukHidalgo OJ, et al. Exploring Anopheles gut bacteria for Plasmodium blocking activity. Environ Microbiol. 2014;16:2980-94

31. Cunha MS, Esposito DL, Rocco IM, Maeda AY, Vasami FG, Nogueira JS, et al. First complete genome sequence of Zika virus (Flaviviridae, Flavivirus) from an autochthonous transmission in Brazil. Genome Announc. 2016;4:e00032-16.

32. Gonçalves CM, Melo FF, Bezerra JM, Chaves BA, Silva BM, Silva LD, et al. Distinct variation in vector competence among nine field populations of Aedes aegypti from a Brazilian dengue-endemic risk city. Parasit Vectors. 2014;7:320.

33. Booth C, Bergan T, Bennett P, Brown A, Colwell R, Craig A, et al. Methods in microbiology. In: Immunology of infection. 2002;32.

34. Lacey LA. Manual of techniques in insect pathology (Biological techniques series). Cambridge: Academic Press; 1997.

35. Altschul SF, Gish W, Miller W, Myers EW, Lipman DJ. Basic local alignment search tool. J Mol Biol. 1990;215:403-10.

36. Wang Y, Qian PY. Conservative fragments in bacterial 16S rRNA genes and primer design for 165 ribosomal DNA amplicons in metagenomic studies. PLOS ONE. 2009;4:e7401.

37. Yang B, Wang Y, Qian PY. Sensitivity and correlation of hypervariable regions in 16S rRNA genes in phylogenetic analysis. BMC Bioinform. 2016;17:135

38. Galkiewicz JP, Kellogg CA. Cross-kingdom amplification using bacteriaspecific primers: complications for studies of coral microbial ecology. Appl Environ Microbiol. 2008;74:7828-31.

39. Sharma U, Singh S. Insect vectors of Leishmania: distribution, physiology and their control. J Vector Borne Dis. 2008;45:255-72. Retraction in: J Vector Borne Dis. 2012;49:54.

40. Liu B, Gibbons T, Ghodsi M, Treangen T, Pop M. Accurate and fast estimation of taxonomic profiles from metagenomic shotgun sequences. BMC Genomics. 2011;12:S4. 
41. Lanciotti RS, Kosoy OL, Laven JJ, Velez JO, Lambert AJ, Johnson AJ, et al. Genetic and serologic properties of Zika virus associated with an epidemic, Yap State, Micronesia, 2007. Emerg Infect Dis. 2008;14:1232-9.

42. Dutra HLC, Rocha MN, Dias FBS, Mansur SB, Caragata EP, Moreira LA. Wolbachia blocks currently circulating Zika virus isolates in Brazilian Aedes aegypti mosquitoes. Cell Host Microbe. 2016;8:771-4.

43. Secundino NFC, Chaves BA, Orfano AS, Silveira KRD, Rodrigues NB, Campolina TB, et al. Zika virus transmission to mouse ear by mosquito bite: a laboratory model that replicates the natural transmission process. Parasit Vectors. 2017;10:346.

44. Rojas-Pinzón PA, Dussán J. Contribution of Lysinibacillus sphaericus hemolysin and chitin- binding protein in entomopathogenic activity against insecticide resistant Aedes aegypti. World J Microbiol Biotechnol. 2017;33:181.

45. Bai L, Wang L, Vega-Rodríguez J, Wang G, Wang S. A gut symbiotic bacterium Serratia marcescens renders mosquito resistance to Plasmodium infection through activation of mosquito immune responses. Front Microbiol. 2019:10:1580.

46. Wu P, Sun P, Nie K, Zhu Y, Shi M, Xiao C, et al. A gut commensal bacterium promotes mosquito permissiveness to arboviruses. Cell Host Microbe. 2019;25:101-112.e5.

47. Campolina TB, Villegas LEM, Monteiro CC, Pimenta PFP, Secundino NFC. Tripartite interactions: Leishmania, microbiota and Lutzomyia longipalpis. PLoS Negl Trop Dis. 2020;14:e0008666.

48. Javed F, Manzoor KN, Ali M, Haq IU, Khan AA, Zaib A, Manzoor S. Zika virus: what we need to know? J Basic Microbiol. 2018;58:3-16.

49. Russell K, Hills SL, Oster AM, Porse CC, Danyluk G, Cone M, et al. Male-tofemale sexual transmission of Zika Virus-United States, january-april 2016. Clin Infect Dis. 2017;64:211-3.

50. Costa LC, Veiga RV, Oliveira JF, Rodrigues MS, Andrade RFS, Paixão ES, et al. New insights on the Zika virus arrival in the Americas and spatiotemporal reconstruction of the epidemic dynamics in Brazil. Viruses. 2020;13:12.

51. Pan American Health Organization/World Health Organization. Cases of Zika virus disease. Washington, D.C: PAHO/WHO; 2020.

52. Lowe R, Barcellos C, Brasil P, Cruz OG, Honório NA, Kuper H, et al. The Zika virus epidemic in Brazil: from discovery to future implications. Int J Environ Res Public Health. 2018;15:96.

53. Muturi EJ, Dunlap C, Ramirez JL, Rooney AP, Kim C-H. Host blood-meal source has a strong impact on gut microbiota of Aedes aegypti. FEMS Microbiol Ecol. 2019. https://doi.org/10.1093/femsec/fiy213.

54. Yadav KK, Bora A, Datta S, Chandel K, Gogoi HK, Prasad GBKS, et al. Molecular characterization of midgut microbiota of Aedes albopictus and Aedes aegypti from Arunachal Pradesh, India. Parasites Vectors. 2015;8:641.

55. Champion $\mathrm{CJ}, \mathrm{Xu}$ J. The impact of metagenomic interplay on the mosquito redox homeostasis. Free Radic Biol Med. 2017;105:79-85.

56. Gusmão DS, Santos AV, Marini DC, Bacci M, Berbert-Molina MA, Lemos FJA. Culture-dependent and culture-independent characterization of microorganisms associated with Aedes aegypti (Diptera: Culicidae) (L.) and dynamics of bacterial colonization in the midgut. Acta Trop. 2010;115:275-81.

57. David MR, dos Santos LMB, Vicente ACP, Maciel-de-Freitas R. Effects of environment, dietary regime and ageing on the dengue vector microbiota: evidence of a core microbiota throughout Aedes aegypti lifespan. Mem Inst Oswaldo Cruz. 2016;111:577-87.

58. Dickson LB, Ghozlane A, Volant S, Bouchier C, Ma L, Vega-Rúa A, et al. Diverse laboratory colonies of Aedes aegypti harbor the same adult midgut bacterial microbiome. Parasit Vectors. 2018;11:207.

59. Ramos-Nino ME, Fitzpatrick DM, Eckstrom KM, Tighe S, Hattaway LM, Hsueh AN, et al. Metagenomic analysis of Aedes aegypti and Culex quinquefasciatus mosquitoes from Grenada, West Indies. PLoS ONE. 2020;15:e0231047.

60. Yadav KK, Datta S, Naglot A, Bora A, Hmuaka V, Bhagyawant S, et al. Diversity of cultivable midgut microbiota at different stages of the Asian tiger tosquito, Aedes albopictus from Tezpur, India. PLoS ONE. 2016;11:e0167409.

61. Chandel K, Mendki MJ, Parikh RY, Kulkarni G, Tikar SN, Sukumaran D, et al. Midgut microbial community of Culex quinquefasciatus mosquito populations from India. PLoS ONE. 2013;8:e80453.

62. Tchioffo MT, Boissière A, Churcher TS, Abate L, Gimonneau G, Nsango SE et al. Modulation of malaria infection in Anopheles gambiae mosquitoes exposed to natural midgut bacteria. PLoS ONE. 2013;8:e81663.
63. Minard G, Mavingui P, Moro CV. Diversity and function of bacterial microbiota in the mosquito holobiont. Parasit Vectors. 2013;6:146.

64. Minard G, Tran FH, Raharimalala FN, Hellard E, Ravelonandro P, Mavingui $P$, et al. Prevalence, genomic and metabolic profiles of Acinetobacter and Asaia associated with field-caught Aedes albopictus from Madagascar. FEMS Microbiol Ecol. 2013;83:63-73.

65. Schumann P, Weiss N, Stackebrandt E. Reclassification of Cellulomonas cellulans (Stackebrandt and Keddie 1986) as Cellulosimicrobium cellulans gen. nov., comb. nov. Int J Syst Evol Microbiol. 2001;51:1007-10.

66. Bakalidou A, Kämpfer P, Berchtold M, Kuhnigk T, Wenzel M, König H. Cellulosimicrobium variabile sp. nov., a cellulolytic bacterium from the hindgut of the termite Mastotermes darwiniensis. Int I Syst Evol Microbiol. 2002;52:1185-92.

67. Valiente Moro C, Tran FH, Nantenaina Raharimalala F, Ravelonandro P, Mavingui P. Diversity of culturable bacteria including Pantoea in wild mosquito Aedes albopictus. BMC Microbiol. 2013;13:70.

68. Guégan M, Zouache K, Démichel C, Minard G, Van Tran V, Potier P, et al. The mosquito holobiont: fresh insight into mosquito-microbiota interactions. Microbiome. 2018;6:49.

69. Hegde S, Khanipov K, Albayrak L, Golovko G, Pimenova M, Saldaña MA, et al. Microbiome interaction networks and community structure from laboratory-reared and field-collected Aedes aegypti, Aedes albopictus, and Culex quinquefasciatus mosquito vectors. Front Microbiol. 2018;9:2160.

70. Agarwal A, Parida M, Dash PK. Impact of transmission cycles and vector competence on global expansion and emergence of arboviruses. Rev Med Virol. 2017;27:e1941.

71. Hardy JL, Houk EJ, Kramer LD, Reeves WC. Intrinsic factors affecting vector competence of mosquitoes for arboviruses. Annu Rev Entomol. 1983;28:229-62.

72. Schneider BS, Higgs S. The enhancement of arbovirus transmission and disease by mosquito saliva is associated with modulation of the host immune response. Trans R Soc Trop Med Hyg. 2008;102:400-8.

73. Styer LM, Kent KA, Albright RG, Bennett CJ, Kramer LD, Bernard KA. Mosquitoes inoculate high doses of West Nile virus as they probe and feed on live hosts. PLoS Pathog. 2007:3:1262-70.

74. Bonica MB, Goenaga S, Martin ML, Feroci M, Luppo V, Muttis E, et al. Vector competence of Aedes aegypti for different strains of Zika virus in Argentina. PLoS Negl Trop Dis. 2019;13:e0007433.

75. Chouin-Carneiro T, David MR, Nogueira FB, Santos FdB, Lourenço-deOliveira R. Zika virus transmission by Brazilian Aedes aegypti and Aedes albopictus is virus dose and temperature-dependent. PLoS Negl Trop Dis. 2020;14:e0008527.

76. Osei-Poku J, Mbogo CM, Palmer WJ, Jiggins FM. Deep sequencing reveals extensive variation in the gut microbiota of wild mosquitoes from Kenya. Mol Ecol. 2012;21:5138-50.

77. Zouache K, Raharimalala FN, Raquin V, Tran-Van V, Raveloson LHR, Ravelonandro $\mathrm{P}$, et al. Bacterial diversity of field-caught mosquitoes, Aedes albopictus and Aedes aegypti, from different geographic regions of Madagascar. FEMS Microbiol Ecol. 2011;75:377-89.

78. Möhlmann TWR, Vogels CBF, Göertz GP, Pijlman GP, ter Braak CJF, te Beest $\mathrm{DE}$, et al. Impact of gut bacteria on the infection and transmission of pathogenic arboviruses by biting midges and mosquitoes. Microb Ecol. 2020;80:703-17.

79. Rosso F, Tagliapietra V, Albanese D, Pindo M, Baldacchino F, Arnoldi D, et al. Reduced diversity of gut microbiota in two Aedes mosquitoes species in areas of recent invasion. Sci Rep. 2018;8:16091.

80. Zouache K, Michelland RJ, Failloux A-B, Grundmann GL, Mavingui P. Chikungunya virus impacts the diversity of symbiotic bacteria in mosquito vector. Mol Ecol. 2012;21:2297-309.

81. Qing W, Zhijing X, Guangfu Y, Fengxia M, Qiyong L, Zhong Z, et al. Variation in the microbiota across different developmental stages of Aedes albopictus is affected by ampicillin exposure. MicrobiologyOpen. 2020;9:e1026.

\section{Publisher's Note}

Springer Nature remains neutral with regard to jurisdictional claims in published maps and institutional affiliations. 